\section{Reforming science in Japan}

SIR - Despite a certain partiality (11 references to Tokyo University in 9 pages), some of the observations made by John Maddox and David Swinbanks in their report on "Science in Japan" (Nature 359, 573-582; 1992) were so ironically succinct as to make us both groan and laugh in affirmation. That the rigidity of the system in Japanese universities leads to inbreeding is illustrated by the fact that in the veterinary faculty of the national university where we work, 13 out of 16 professors and all 16 of the associate professors have received at least one degree at this university. No women are employed as staff members, no true postdoctoral positions are available, and one third of this year's graduates will remain for another four years to do their PhD. The number of laboratory technicians has dropped in the past 20 years (currently only one is employed), and grant conditions usually make it impossible to hire them. There are, however, some encouraging signs: two years ago the faculty made its first-ever appointment of a non-Japanese (albeit a graduate) to a staff position.

Twenty years ago in the Nature special issue on Japan, attention was drawn to criticisms of problems as well as to overbearing administration and defects of the Monbusho grant system. These problems are all alive and well in Japanese universities today. But one wonders if these conditions are really amenable to reform by policy or decree, or whether, like many of Japan's characteristics, they are a product of compromise between the desire for stability and the necessity for improvement. Any type of reform would be more likely to involve consensus and consultation at all levels, a slow process but one that usually receives support from the majority of the people affected. It must be remembered that Japan has made significant contributions to science despite its shortcomings. The attention to detail, care and diligence of Japanese researchers will ensure that these people will continue to produce top class research, and hopefully to be given the recognition their efforts deserve.

Matthew C. Playford

Masao Kamiya

Department of Veterinary Parasitology, Hokkaido University,

Sapporo, Japan

SIR - We appreciate the attention that Nature pays to Tokyo University, but in the News article $(\mathbf{3 9 0}, 403 ; 1992)$ about the external evaluation of one of our departments there are two points on which we believe our motives have been misinterpreted.
The first concerns the white paper or policy document (hakusho in Japanese), a 630-page report published by the university. Because of its length, the report was embargoed until 7 December to give the press time to digest it. For the same reason, we organized a general lecture on 27 November followed by a press conference on 4 December. Regrettably, Nature published comments on the contents of the hakusho on 3 December, before the embargo was lifted.

The second point concerns the content. You quote one of the deans of Tokyo University as saying that the hakusho is largely "propaganda" and contains little "true evaluation". However, the dean himself has explained that his argument was rather that the hakusho is a report of our internal study, which will be followed by an independent external evaluation. In other words, the hakusho is the first necessary step to "true evaluation". The complex nature of the task of this internal evaluation demands that we inform people of the process through the contents of the hakusho.

M. Aoyagi

(Chairman)

Committee of Public Relations,

University of Tokyo,

7-3-1 Hongo, Bunkyo-ku,

Tokyo 113, Japan

Nature did not take part in the meetings of 27 November and 4 December and received no material from them. We learned of the hakusho independently from several sources. - Editor, Nature.

\section{Irradiated graphite}

SIR - In 1949, I was sent from the Atomic Energy Research Establishment, Harwell, to the Chalk River Laboratory, Canada, to study the possible changes in the physical dimensions of graphite under irradiation in the NRX nuclear reactor. The main objective was to confirm the existence of a dimensional effect and establish a quantitative relationship between exposure to radiation and dimensional change ${ }^{1}$.

After accumulating some graphite samples with considerable exposures, my colleagues and I did some heating experiments to see whether the effect could be annealed out. Clearly, some recovery took place at 100,200 and particularly $250^{\circ} \mathrm{C}$. In one case the oven temperature appeared to rise much higher than it should have done, possibly higher than the oven design limit; the sample had annealed much more than the others. At this stage we assumed that the oven thermostat or thermocouple had failed. We were not able to continue these tests because only a few highly irradiated samples were available.

We believed that the effect we were measuring resulted from increasing disorder in the crystal structure caused by carbon atoms being knocked out of their lattice positions by fast neutrons into interstitial positions between the hexagon layers characteristic of graphite. Presumably, the displaced atoms are in positions of greater potential energy. We used the term 'Wigner effect' to describe the dimensional changes. I was not then aware of the significance of stored energy in graphite (later referred to as the Wigner effect). In 1953, it was appreciated that graphite-moderated reactors could store energy in the graphite, following an accidental release in one of the Windscale (now Sellafield) reactors. Following this, the stored energy was released in a controlled way as part of the routine operating procedures.

In October 1957, a controlled release in one of the Windscale reactors went seriously wrong, resulting in a fire in the core, which was destroyed. Subsequently, the graphite research programme was considerably extended.

It seems possible that in our anomalous annealing measurement in 1949-51 we were observing the release of stored energy in the graphite sample. Had we followed our preliminary measurement with more studies, we might have understood the properties of energy storage and release several years before the Windscale accident. Such a view, of course, has the benefit of hindsight.

\section{N. J. Pattenden}

73B Essex Street,

Newbury, Berkshire RG14 6RA, UK

1. Sheard, H. \& Pattenden, N. J. The Effect of Pile Irradiation on the Linear Dimensions of AGXP Graphite. Chalk River report CRNE-496, 1952.

\section{CEA research}

SIR - Declan Butler's statement that "research in plant physiology will be phased out during the next three years" at the Commissariat à l'Energie Atomique (CEA, Nature 359, 568; 1992) has caused some concern to researchers here.

In fact, only microalgae biotechnology and radioagronomy are to be phased out in the coming year; research on plant ionization will concentrate on fundamentals and will be expertly appraised; plant physiology and research on artificial ecosystems will remain a priority, as will protein engineering.

\section{Michel Suscillon}

Commissariat à l'Energie Atomique,

31-33 Rue de la Fédération,

75752 Paris Cedex 15, France 